\section{P96 Process for Applying the FA0's School Food and Nutrition Education Capacity Assessment Tool in the Context of COVID-19: Results from El Salvador}

Luisa Fernanda Lopez-Hernandez, MS, Food Agriculture Organization of the United Nations (FAO); Melissa Vargas, MS, Food and Agriculture Organization of the United Nations (FAO); Yenory Hernández-Garbanzo, PhD, Food and Agriculture Organization of the United Nations (FAO); Jorge Ulises Gonzalez, MS, Food and Agriculture Organization of the United Nations (FAO); Israel RíosCastillo,PhD, israel.rios@fao.org, Food and Agriculture Organization of the United Nations (FAO), Edificio 238, $3^{\circ}$ Planta, Ciudad del Saber, Clayton, Ciudad de Panamá, Panamá, 7114; Fatima Hachem, PhD, Food and Agriculture Organization of the United Nations (FAO); Reina OsorioGuevara, BS, Food and Agriculture Organization of the United Nations (FAO)

Background: Due to the nature of effective school food and nutrition education (SFNE) and the recent emphasis on it, FAO has developed a tool to assist member countries assess capacities, strengths, and gaps for developing and implementing successful SFNE programs at national and/ or subnational level.

Objective: To describe the process used for applying FAO's SFNE Capacity Assessment Tool in El Salvador.

Study Design, Setting, Participants: A cross-sectional qualitative study conducted through the following 5 phases: 1) setting up an FAO's facilitation team; 2) a SFNE stakeholder mapping ( $\mathrm{n}=15$, from government/NGOs staff to teachers and families); 3) background research of national secondary data, policies, reports, and materials relevant to SFNE; 4) interviews with key actors $(n=7)$ and facilitation of multi-stakeholder consultative workshops $(\mathrm{n}=5)$; and 5) report and follow-up.

Measurable Outcome/Analysis: The tool comprises a series of guiding questions to assess individual SFNE capacities, as well as organizational capacity and the policy environment. Key variables of interest comprised policy frameworks, political commitment, program-related capacities, school system, individual training schemes, among others. Descriptive content analysis was carried out to synthesize the results from each dimension.

Results: Main capacity gaps identified were: weak institutional coherence, exclusion of SFNE in the formal school curriculum, lack of opportunities to engage all schoollevel stakeholders in learning activities, and insufficient educational materials for all schools. Key strengths focused on the inclusion of SFNE into key policies, and the availability of SFNE training schemes for front-line educators. Prioritized proposed solutions centered around advocacy for the allocation of SFNE-specific government budget, leveraging on existing multisector platforms to enhance institutional coherence, and strengthening of training of curriculum developers.

Conclusion: The application of the tool evidenced key SFNE capacity gaps that needed to be addressed at national level, and provided a clear and nationally-endorsed roadmap to enhance the impact of SFNE in El Salvador.
Funding: Food Agriculture Organization of the United Nations (FAO).

\section{P97 Reducing Obesogenic Behaviors Through a Culturally-Tailored Family-Based Program: "Abriendo Caminos"}

Maribel Barragan, BS, RD, University of Illinois at Urbana Champaign; Viridiana Luna, MS, BS, Illinois Action for Children; Amber Hammons, PhD, California State University, Fresno; Norma Olvera, PhD, University of Houston; Kimberly Greder, PhD, Iowa State University; Margarita Teran-Garcia, PhD, MD, teranmd@illinois.edu, University of Illinois at Urbana Champaign, $904 \mathrm{~W}$ Nevada, Urbana, IL, 61801

Introduction: Hispanic children in the U.S. exhibit the highest rates of obesity compared to other racial/ethnic groups. Effective interventions to reduce the burden of this growing epidemic are needed. Obesity prevention interventions can improve weight outcomes by targeting modifiable lifestyle factors such as dietary patterns and physical activity. Furthermore, there is evidence that culturally-tailored interventions increase fruit and vegetable consumption and decrease sugar-sweetened beverage (SSB) consumption among Hispanic children.

Objective: To evaluate the effect of Abriendo Caminos 2 (AC2) on Hispanic children's dietary behaviors.

Methods: AC2 is a multi-state randomized-control trial that aims to prevent childhood obesity among Hispanic families by providing culturally-tailored nutrition, physical activity, and family wellness education. Families of Mexican or Puerto Rican origin with a child between the ages of 6-18 years were recruited from Illinois, California, Iowa, and Texas. Parents' reported their child's dietary intake of SSB, fruit juice, fruits, French fries, vegetables, fast food, sweets, and salty snacks using items from the U.S. Department of Education's Early Childhood Longitudinal Study, Birth Cohort protocol. Pre/post dietary changes were evaluated using Generalized Estimating Equation models adjusted for site, child sex, age, and mother's education using SAS 9.4 (SAS Institute, Cary, NC, USA).

Results: Children randomized to the intervention arm reduced their consumption of SSB (OR 0.35, 95\% CI 0.15 , $0.84, P=0.003$ ), fast food (OR $0.53,95 \%$ CI $0.30,0.92, P=$ 0.03 ), and increased consumption of vegetables (OR 1.95, $95 \%$ CI $1.13,3.36, P=0.02$ ) after 6 weeks of participating in the program. Children in the control group reportedly decreased SSB consumption frequency (OR 0.40, 95\% CI $0.17,0.97, P=0.04)$. There were no changes in the frequency of consumption of the other food items for either group.

Conclusion: Culturally-tailored family-based interventions can help improve dietary behaviors among Hispanic children. Future research should address methods to help Hispanic children transition short-term changes into lifestyle habits.

Funding: USDA. 